\title{
Sources of organic aerosols in Europe: A modelling study using CAMx with modified volatility basis set scheme
}

Jianhui Jiang ${ }^{1}$, Sebnem Aksoyoglu ${ }^{1}$, Imad El-Haddad ${ }^{1}$, Giancarlo Ciarelli ${ }^{2}$, Hugo A. C. Denier van der 5 Gon $^{3}$, Francesco Canonaco ${ }^{1}$, Stefania Gilardoni ${ }^{4}$, Marco Paglione $^{4, *}$, María Cruz Minguillón ${ }^{5}$, Olivier Favez $^{6}$, Yunjiang Zhang ${ }^{6,7}$, Nicolas Marchand ${ }^{8}$, Liqing Hao ${ }^{9}$, Annele Virtanen ${ }^{9}$, Kalliopi Florou ${ }^{10}$, Colin $\mathrm{O}^{\prime}$ Dowd $^{11}$, Jurgita Ovadnevaite ${ }^{11}$, Urs Baltensperger ${ }^{1}$, and André S. H. Prévôt ${ }^{1}$

${ }^{1}$ Laboratory of Atmospheric Chemistry, Paul Scherrer Institute, 5232 Villigen PSI, Switzerland

${ }^{2}$ Department of Chemical Engineering, Carnegie Mellon University, Pittsburgh, USA

$10 \quad{ }^{3}$ TNO, Department of Climate, Air and Sustainability, Utrecht, the Netherlands

${ }^{4}$ Italian National Research Council - Institute of Atmospheric Sciences and Climate, Bologna, Italy

${ }^{5}$ Institute of Environmental Assessment and Water Research (IDAEA), CSIC, 08034 Barcelona, Spain

${ }^{6}$ Institut National de l'Environnement Industriel et des Risques (INERIS), Verneuil-en-Halatte, France

${ }^{7}$ Laboratoire des Sciences du Climat et de l'Environnement (LSCE), Gif-sur-Yvette, France

$15{ }^{8}$ Aix-Marseille Univ, CNRS, LCE, Marseille, France

${ }^{9}$ Department of Applied Physics, University of Eastern Finland, P.O. Box 1627, FI-70211 Kuopio, Finland

${ }^{10}$ Department of Chemical Engineering, University of Patras, 26500 Patras, Greece

${ }^{11}$ School of Physics, Ryan Institute's Centre for Climate and Air Pollution Studies, and Marine Renewable Energy Ireland, National University of Ireland Galway, University Road, Galway, H91 CF50, Ireland

20 "now at: Institute of Chemical Engineering Sciences, Foundation for Research and Technology Hellas (FORTH/ICE-HT), Patras, Greece

Correspondence to: Sebnem Aksoyoglu (sebnem.aksoyoglu@ psi.ch), Jianhui Jiang (jianhui.jiang@psi.ch) 
Table S1 Description of semi-volatile organic compounds (SVOC) and intermediate-volatility organic compounds (IVOC). The same calculations were adopted for IVOC emissions in BASE and NEW. GV: Gasoline Vehicles; DV: Diesel Vehicles; BB: Biomass Burning; OthA: Other anthropogenic sources.

\begin{tabular}{|c|c|c|c|c|c|}
\hline \multirow{2}{*}{ Species } & \multirow{2}{*}{ Source } & \multicolumn{2}{|c|}{ Calculations } & \multirow{2}{*}{ References } & \multirow{2}{*}{ Descriptions } \\
\hline & & BASE & NEW & & \\
\hline SVOC & $\begin{array}{l}\text { GV } \\
\text { DV } \\
\text { BB } \\
\text { OthA }\end{array}$ & $\begin{array}{l}=\text { POA_GV } \\
=\text { POA_DV } \\
=\text { POA_BB } \\
=\text { POA_OthA }\end{array}$ & $\begin{array}{l}=3 * \text { POA_GV } \\
=3 * \text { POA_DV } \\
=3 * \text { POA_BB } \\
=3 * \text { POA_OthA }\end{array}$ & $\begin{array}{l}\text { Shrivastava et al., 2011; } \\
\text { Tsimpidi et al., 2010; } \\
\text { Ciarelli et al., 2017a }\end{array}$ & $\begin{array}{l}\text { POA emissions of each source } \\
\text { were calculated from TNO } \mathrm{PM}_{2.5} \\
\text { emissions }\end{array}$ \\
\hline IVOC & $\begin{array}{l}\text { GV } \\
\text { DV } \\
\text { BB } \\
\text { OthA }\end{array}$ & $\begin{array}{l}=25 \% * \text { NMV } \\
=20 \% * \text { NMV } \\
=4.5 * \text { POA_E } \\
=1.5 * \text { POA_C }\end{array}$ & $\begin{array}{l}\text { DC_GV } \\
\text { BC_DV } \\
\text { B thA }\end{array}$ & $\begin{array}{l}\text { Jathar et al., } 2014 \\
\text { Jathar et al., } 2014 \\
\text { Ciarelli et al., } 2017 \\
\text { Robinson et al., } 2007\end{array}$ & $\begin{array}{l}\text { The portion of NMVOCs } \\
\text { considered as IVOCs ( } 25 \% \text { for } \\
\text { GV, } 20 \% \text { for DV) were removed } \\
\text { from the NMVOC emissions }\end{array}$ \\
\hline
\end{tabular}


Table S2. Evaluation of the meteorological parameters in winter (February, number of stations = 1094) and summer (July, number of stations $=753$ ). Performance criteria for model results are from Emery et al., (2001). MB: mean bias; MGE: mean gross error; RMSE: root-mean-square error; IOA: index of agreement.

\begin{tabular}{ccccccccccccc}
\hline $\begin{array}{c}\text { Meteorological } \\
\text { parameters }\end{array}$ & \multicolumn{3}{c}{ MB } & \multicolumn{3}{c}{ MGE } & \multicolumn{3}{c}{ RMSE } & \multicolumn{3}{c}{ IOA(-) } \\
\cline { 2 - 12 }$y$ & Feb. & Jul. & Criteria & Feb. & Jul. & Criteria & Feb. & Jul. & Criteria & Feb. & Jul. & Criteria \\
\hline $\begin{array}{c}\text { Temperature } \\
\left({ }^{\circ} \mathrm{C}\right)\end{array}$ & -1.0 & -0.1 & $\leq \pm 0.5$ & 1.2 & 0.7 & $\leq \pm 2$ & 1.7 & 0.9 & - & 1.0 & 1.0 & $\geq 0.8$ \\
$\begin{array}{c}\text { Wind speed } \\
\left(\mathrm{m} \mathrm{s}^{-1}\right)\end{array}$ & -0.1 & -0.3 & $\leq \pm 0.5$ & 1.3 & 0.9 & - & 1.6 & 1.2 & $\leq 2$ & 0.8 & 0.5 & $\geq 0.6$ \\
$\begin{array}{c}\text { Wind direction } \\
\left({ }^{\circ}\right)\end{array}$ & 5.2 & 15.8 & $\leq \pm 10$ & 18.1 & 22.6 & $\leq 30$ & 28.4 & 33.3 & - & 0.7 & 0.8 & - \\
$\begin{array}{c}\text { Humidity } \\
\left.(\mathrm{g} \mathrm{kg})^{-1}\right)\end{array}$ & -0.1 & 0.0 & $\leq \pm 1$ & 0.2 & 0.4 & $\leq 2$ & 0.3 & 0.5 & - & 1.0 & 1.0 & $\geq 0.6$ \\
$\begin{array}{c}\text { Precipitation } \\
(\mathrm{mm})\end{array}$ & -0.3 & -0.4 & - & 0.3 & 0.5 & - & 0.7 & 1.0 & - & 0.2 & 0.4 & - \\
\hline
\end{tabular}


Table S3. Evaluation of the model performance for the chemical species in winter (February) and summer (July). MB: mean bias; MGE: mean gross error; RMSE: root-mean-square error; MFB: mean fractional bias; MFE: mean fractional error; IOA: index of agreement.

\begin{tabular}{|c|c|c|c|c|c|c|c|c|c|c|c|c|c|c|}
\hline & \multirow{2}{*}{ Model } & \multirow{2}{*}{$\begin{array}{c}\text { Number of } \\
\text { Stations }\end{array}$} & \multicolumn{2}{|c|}{$\mathrm{MB}^{\mathrm{a}}$} & \multicolumn{2}{|c|}{$\mathrm{MGE}^{\mathrm{a}}$} & \multicolumn{2}{|c|}{$\operatorname{MFB}(\%)$} & \multicolumn{2}{|c|}{ MFE (\%) } & \multicolumn{2}{|c|}{ RMSE $^{\mathrm{a}}$} & \multicolumn{2}{|c|}{$\operatorname{IOA}(-)$} \\
\hline & & & Feb. & Jul. & Feb. & Jul. & Feb. & Jul. & Feb. & Jul. & Feb. & Jul. & Feb. & Jul. \\
\hline \multirow[t]{2}{*}{$\mathrm{PM}_{2.5}$} & BASE & 565 & 0.5 & -1.3 & 8.9 & 2.8 & 9 & -12 & 35 & 28 & 11.7 & 3.7 & 0.6 & 0.4 \\
\hline & NEW & 565 & 2.9 & -0.8 & 9.3 & 2.6 & 17 & -7 & 36 & 26 & 11.9 & 3.6 & 0.6 & 0.5 \\
\hline $\mathrm{O}_{3}$ & NEW & 608 & 2.0 & 0.9 & 6.1 & 4.7 & 8.8 & 4.1 & 23.0 & 13.5 & 8.5 & 6.3 & 0.7 & 0.7 \\
\hline $\mathrm{NO}_{2}$ & NEW & 3036 & -6.2 & -2.9 & 8.1 & 5.1 & -43 & -36 & 58.6 & 63.2 & 10.9 & 7.5 & 0.6 & 0.5 \\
\hline $\mathrm{SO}_{2}$ & NEW & 1979 & 6.7 & 3.9 & 7.7 & 4.5 & 77.0 & 65.5 & 98.5 & 98.2 & 17.9 & 10.2 & 0.1 & 0.1 \\
\hline
\end{tabular}

${ }^{\mathrm{a}}$ Units are ppb, except for $\mathrm{PM}_{2.5}$ which is $\mu \mathrm{g} \mathrm{\textrm {m } ^ { - 3 }}$.

5

Table S4. Performance criteria and goals for model results on $\mathrm{PM}_{2.5}$ and ozone (Boylan and Russell, 2006; EPA, 2007).

\begin{tabular}{llll}
\hline Species & Metric & Criteria & Goal \\
\hline $\mathrm{PM}_{2.5}$ & MFB & $\leq \pm 60 \%$ & $\leq \pm 30 \%$ \\
& MFE & $\leq 75 \%$ & $\leq 50 \%$ \\
$\mathrm{O}_{3}$ & MFB & $\leq \pm 30 \%$ & $\leq \pm 15 \%$ \\
& MFE & $\leq 45 \%$ & $\leq 30 \%$ \\
\hline
\end{tabular}

10 
Table S5: Relative contributions (\%) of different sources to the organic aerosol (OA) concentration on a country scale. DJF: December - January - February; JJA: June - July - August.

\begin{tabular}{|c|c|c|c|c|c|c|c|c|c|c|}
\hline \multirow{2}{*}{ Country } & \multicolumn{2}{|c|}{ Gasoline vehicles } & \multicolumn{2}{|c|}{ Diesel vehicles } & \multicolumn{2}{|c|}{ Biomass burning } & \multicolumn{2}{|c|}{ Other anthropogenic } & \multicolumn{2}{|c|}{ Biogenic } \\
\hline & DJF & JJA & DJF & JJA & DJF & JJA & DJF & JJA & DJF & JJA \\
\hline Albania & 2.0 & 6.3 & 1.8 & 3.1 & 71.8 & 34.6 & 9.2 & 21.4 & 15.2 & 34.7 \\
\hline Austria & 1.6 & 4.8 & 3.9 & 3.7 & 59.9 & 15.9 & 10.0 & 18.7 & 24.6 & 57.0 \\
\hline Belarus & 0.9 & 2.2 & 2.1 & 1.7 & 65.7 & 21.1 & 8.4 & 11.5 & 22.8 & 63.5 \\
\hline Belgium & 1.4 & 3.9 & 7.3 & 6.7 & 51.0 & 16.7 & 14.4 & 28.7 & 25.9 & 44.0 \\
\hline Bosnia_and_Herzegovina & 1.3 & 5.5 & 1.4 & 3.2 & 79.8 & 35.4 & 6.4 & 19.8 & 11.2 & 36.1 \\
\hline Bulgaria & 1.4 & 4.0 & 1.4 & 2.3 & 77.9 & 39.2 & 7.9 & 17.5 & 11.3 & 37.0 \\
\hline Croatia & 1.9 & 6.1 & 2.4 & 3.7 & 70.8 & 28.8 & 7.6 & 21.1 & 17.2 & 40.3 \\
\hline Cyprus & 1.3 & 2.5 & 2.5 & 1.5 & 35.6 & 13.5 & 6.1 & 8.9 & 54.6 & 73.6 \\
\hline Czech_Republic & 1.1 & 3.3 & 4.7 & 4.4 & 54.9 & 16.1 & 6.7 & 15.4 & 32.6 & 60.7 \\
\hline Denmark & 0.6 & 3.5 & 2.3 & 2.9 & 64.2 & 20.1 & 8.2 & 25.7 & 24.6 & 47.9 \\
\hline Estonia & 0.4 & 2.0 & 1.1 & 1.3 & 78.5 & 19.1 & 4.3 & 11.1 & 15.8 & 66.5 \\
\hline Finland & 0.4 & 1.4 & 1.8 & 0.9 & 57.0 & 7.8 & 7.6 & 7.4 & 33.2 & 82.5 \\
\hline France & 1.4 & 4.6 & 4.5 & 4.9 & 62.7 & 22.3 & 10.8 & 29.4 & 20.6 & 38.8 \\
\hline Germany & 1.3 & 3.8 & 4.2 & 3.6 & 46.6 & 12.5 & 10.3 & 19.4 & 37.5 & 60.8 \\
\hline Greece & 2.3 & 5.1 & 1.4 & 1.8 & 58.3 & 23.2 & 9.9 & 16.9 & 28.1 & 53.0 \\
\hline Hungary & 1.5 & 4.7 & 2.4 & 3.8 & 74.5 & 30.7 & 8.1 & 20.3 & 13.6 & 40.5 \\
\hline Ireland & 0.6 & 1.6 & 3.2 & 2.8 & 16.0 & 5.5 & 5.8 & 11.2 & 74.4 & 79.0 \\
\hline Italy & 4.4 & 10.9 & 4.4 & 4.5 & 70.0 & 25.2 & 10.5 & 29.3 & 10.7 & 30.1 \\
\hline Latvia & 0.4 & 2.2 & 1.4 & 1.7 & 80.1 & 23.3 & 4.3 & 12.2 & 13.9 & 60.6 \\
\hline Lithuania & 0.6 & 2.6 & 2.8 & 2.5 & 69.3 & 22.2 & 7.5 & 14.9 & 19.8 & 57.8 \\
\hline Luxembourg & 1.6 & 3.9 & 12.9 & 11.6 & 50.3 & 15.5 & 13.3 & 23.6 & 21.9 & 45.5 \\
\hline Northern Macedonia & 1.5 & 4.9 & 1.2 & 2.5 & 73.8 & 36.9 & 7.6 & 18.2 & 15.8 & 37.5 \\
\hline Malta & 5.4 & 11.7 & 3.2 & 6.1 & 57.3 & 20.2 & 19.6 & 45.7 & 14.5 & 16.3 \\
\hline The Netherlands & 1.6 & 3.9 & 7.1 & 5.5 & 44.4 & 11.6 & 14.5 & 28.3 & 32.4 & 50.7 \\
\hline Norway & 0.4 & 1.4 & 1.2 & 1.1 & 59.3 & 9.8 & 9.8 & 11.3 & 29.3 & 76.5 \\
\hline Poland & 0.9 & 3.1 & 5.4 & 4.6 & 56.9 & 17.0 & 8.9 & 17.1 & 27.8 & 58.2 \\
\hline Portugal & 0.9 & 2.2 & 2.4 & 2.8 & 42.4 & 17.8 & 8.9 & 17.9 & 45.4 & 59.3 \\
\hline Republic_of_Moldova & 1.4 & 3.7 & 1.3 & 2.2 & 78.7 & 44.3 & 9.5 & 19.5 & 9.1 & 30.3 \\
\hline Romania & 1.1 & 3.7 & 1.0 & 2.2 & 82.2 & 42.5 & 6.7 & 17.2 & 9.1 & 34.3 \\
\hline Russia & 0.7 & 1.4 & 1.7 & 1.0 & 62.9 & 13.9 & 8.8 & 8.0 & 25.8 & 75.7 \\
\hline Serbia_and_Montenegro & 1.4 & 5.1 & 1.3 & 3.0 & 81.9 & 44.0 & 7.0 & 19.0 & 8.3 & 28.9 \\
\hline Slovakia & 0.9 & 3.8 & 2.3 & 4.0 & 74.1 & 27.5 & 5.3 & 16.8 & 17.4 & 47.9 \\
\hline Slovenia & 1.3 & 5.5 & 2.3 & 3.7 & 76.2 & 26.5 & 5.2 & 17.6 & 14.9 & 46.6 \\
\hline Spain & 0.9 & 2.0 & 2.4 & 2.0 & 42.0 & 18.9 & 8.0 & 17.2 & 46.6 & 59.9 \\
\hline Sweden & 0.5 & 1.6 & 1.5 & 1.0 & 34.3 & 7.0 & 8.8 & 9.7 & 55.0 & 80.6 \\
\hline Switzerland & 2.9 & 6.9 & 4.6 & 3.9 & 56.4 & 17.5 & 15.8 & 23.4 & 20.3 & 48.3 \\
\hline Turkey & 1.1 & 2.3 & 1.2 & 1.3 & 60.7 & 21.2 & 9.0 & 11.9 & 28.0 & 63.3 \\
\hline Ukraine & 1.2 & 3.1 & 1.6 & 2.0 & 71.7 & 33.8 & 11.2 & 17.4 & 14.3 & 43.8 \\
\hline United_Kingdom & 0.8 & 2.0 & 3.7 & 3.0 & 28.1 & 7.5 & 11.5 & 20.7 & 56.0 & 66.7 \\
\hline
\end{tabular}




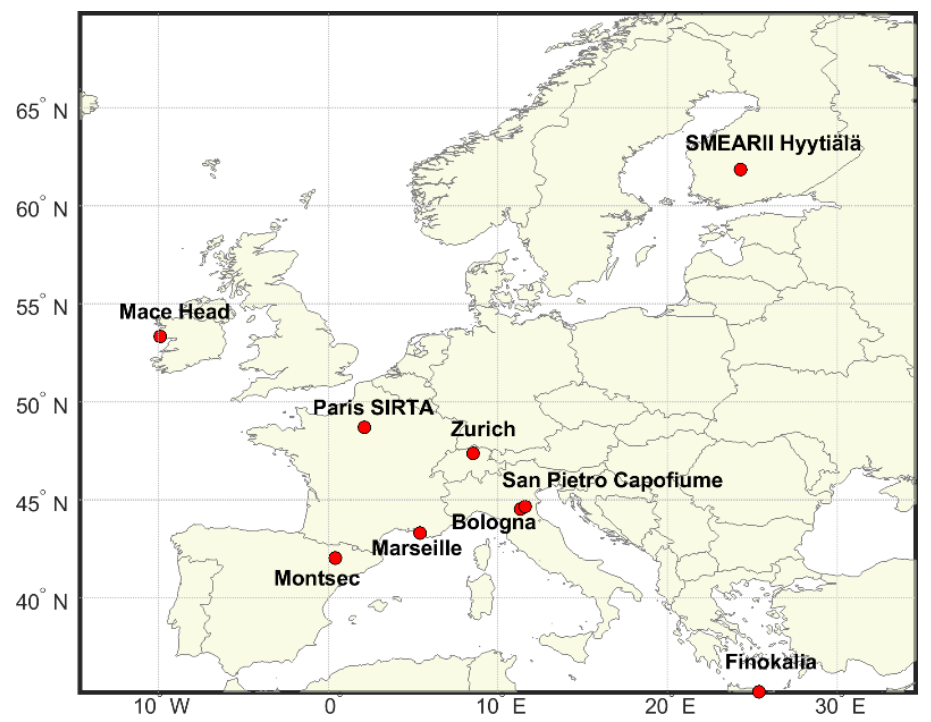

Figure S1: Model domain and spatial distribution of the ACSM/AMS stations. 
(a)
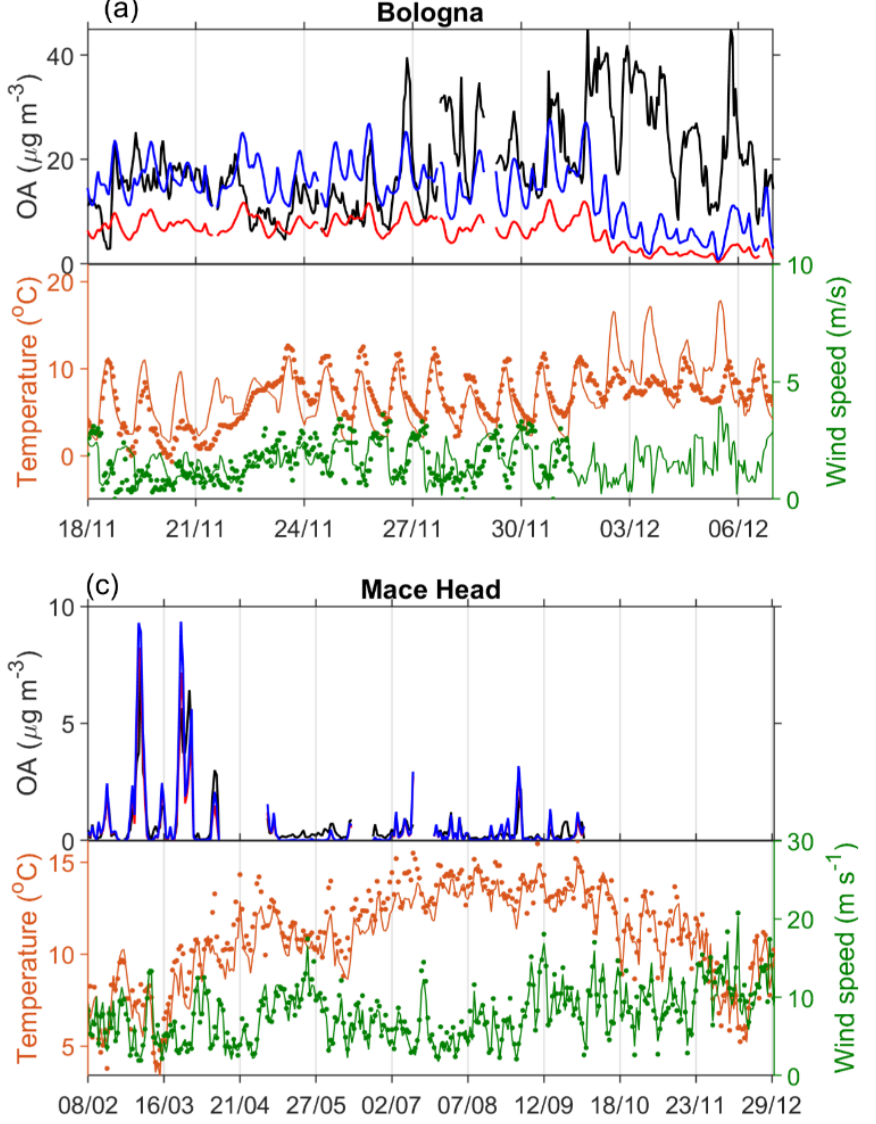

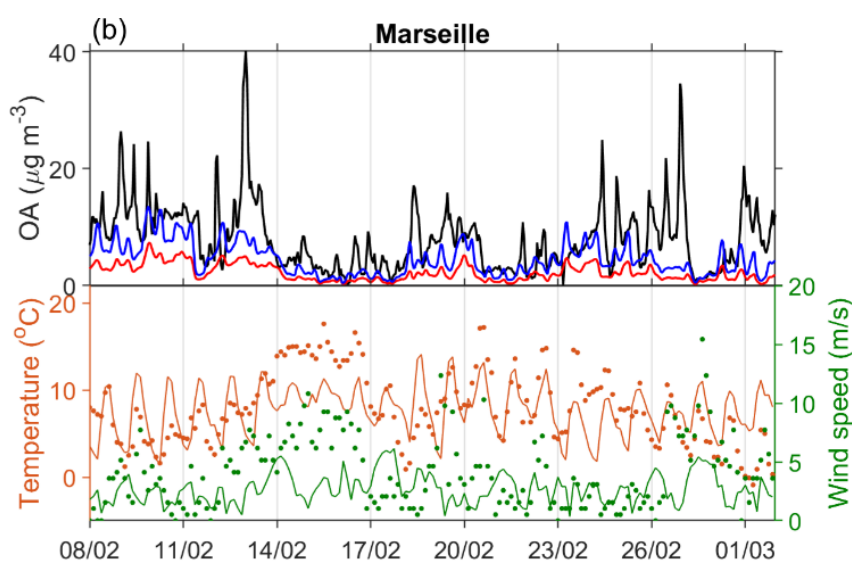

$\begin{array}{ll}\text { - } & \text { Modelled OA - BASE } \\ - & \text { Modelled OA - NEW } \\ - & \text { Measured OA } \\ \text { - } & \text { Observed } \\ & \text { Meteorology } \\ - & \text { Modelled } \\ & \text { Meteorology }\end{array}$

Figure S2: Temporal variations of modelled and measured organic aerosol concentrations together with some meteorological 5 parameters available at Bologna, Marseille and Mace Head. 

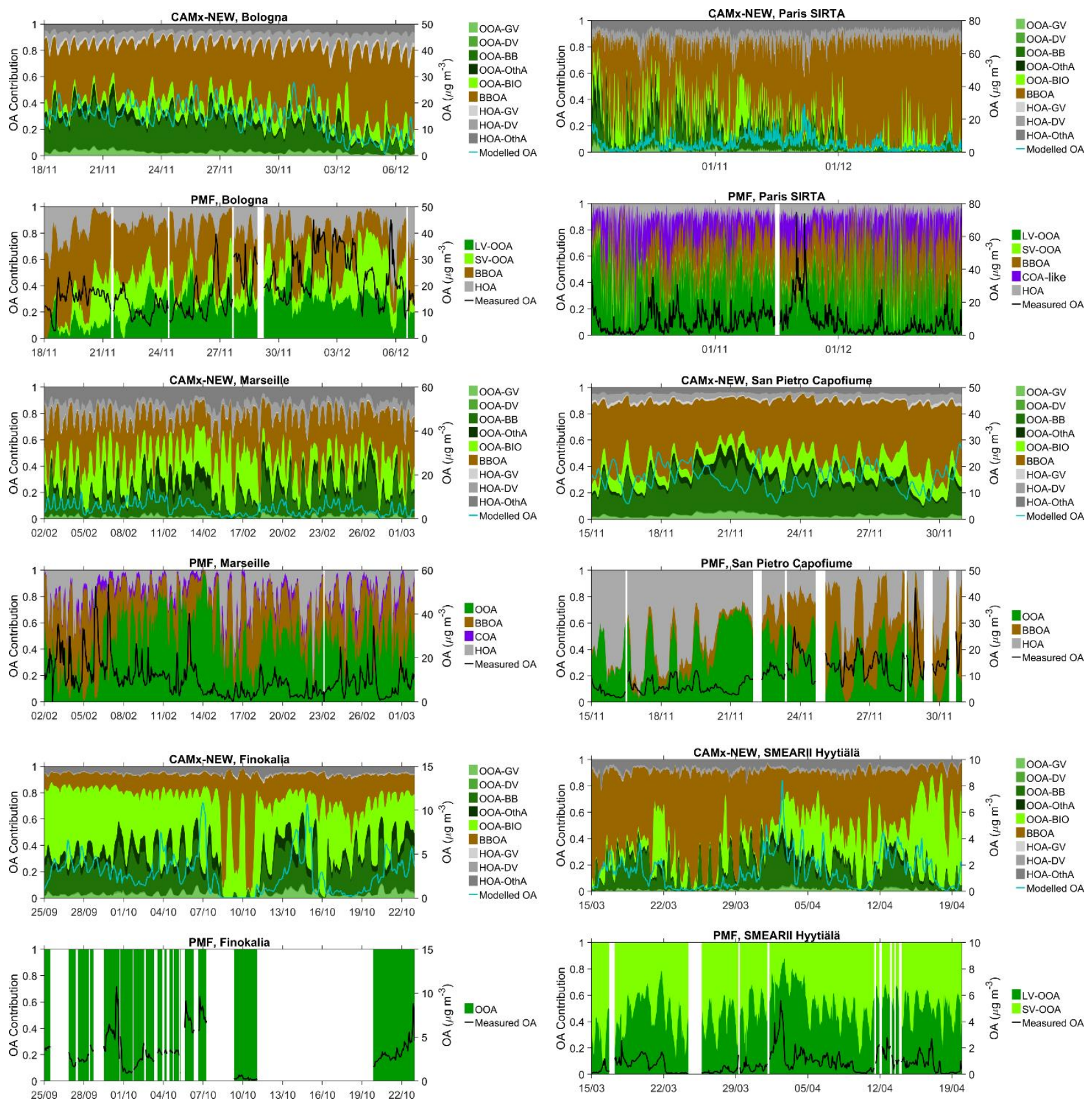

Figure S3: Comparison between modelled relative contribution of OA components and positive matrix factorization (PMF)

5 analysis results. GV: Gasoline Vehicles; DV: Diesel Vehicles; BB: Biomass Burning; OthA: Other anthropogenic sources; BIO: Biogenic sources. 

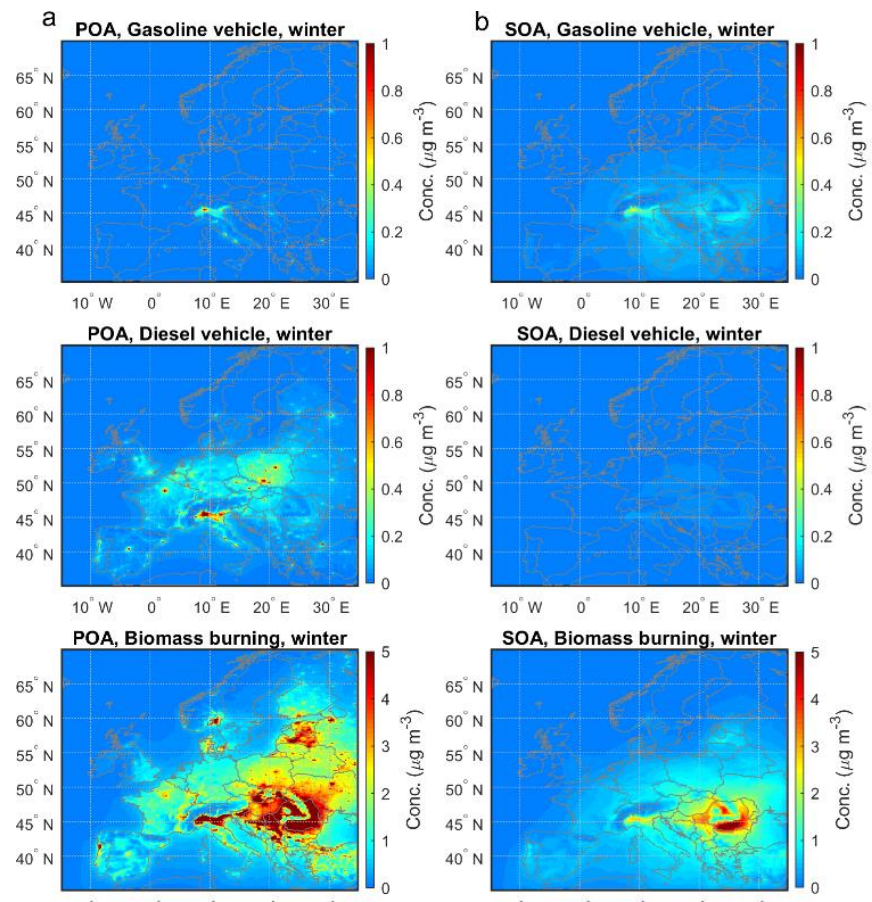

$10^{\circ} \mathrm{W} \quad 0^{\circ} \quad 10^{\circ} \mathrm{E} \quad 20^{\circ} \mathrm{E} \quad 30^{\circ} \mathrm{E}$
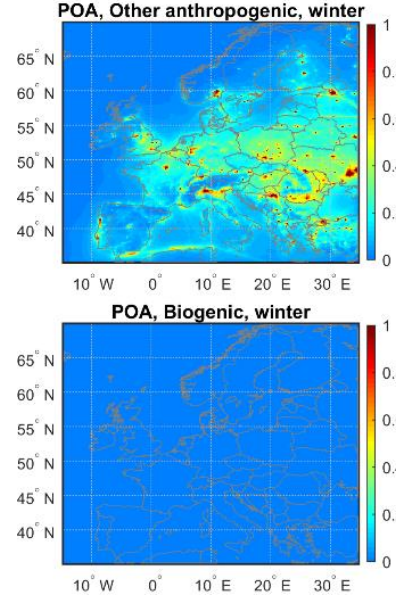

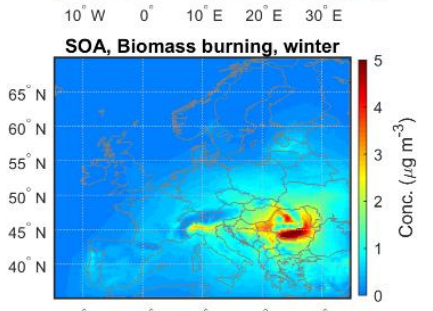

$10^{\circ} \mathrm{W} \quad 0^{\circ} \quad 10^{\circ} \mathrm{E} \quad 20^{\circ} \mathrm{E} \quad 30^{\circ} \mathrm{E}$ SOA, Other anthropogenic, winter

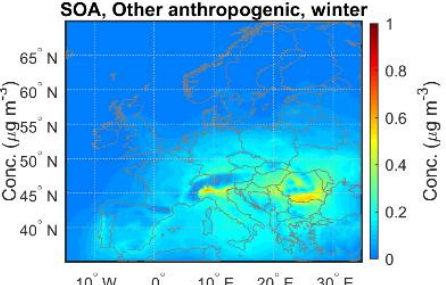

SOA, Biogenic, winter

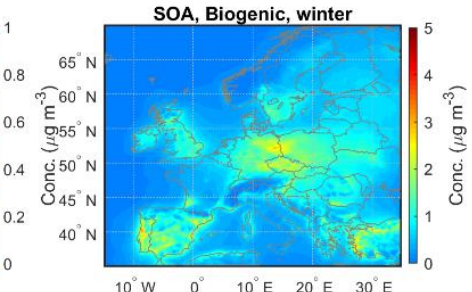

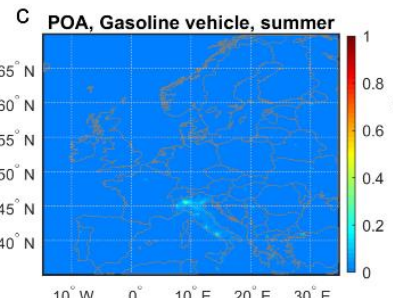

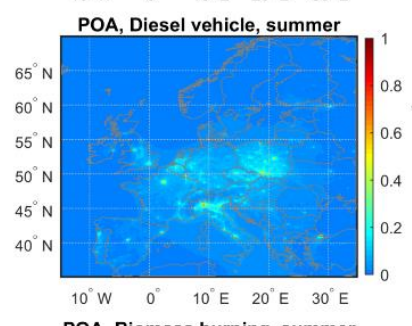

POA, Biomass burning, summer

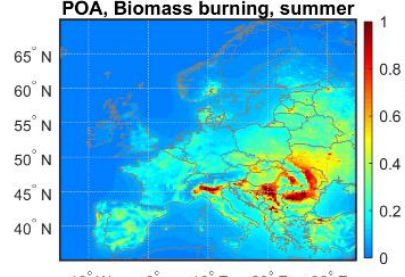

$10^{\circ} \mathrm{W} \quad 0^{\circ} \quad 10^{\circ} \mathrm{E} \quad 20^{\circ} \mathrm{E} \quad 30^{\circ} \mathrm{E}$
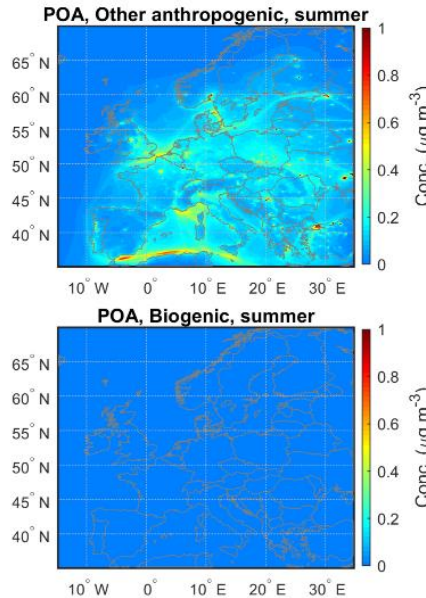

d sOA, Gasoline vehicle, summer

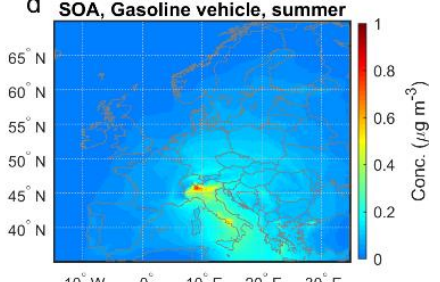

SOA, Diesel vehicle, summer

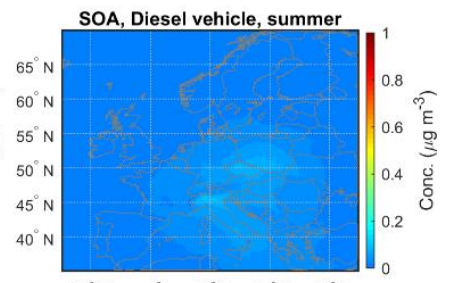

$10^{\circ} \mathrm{W} \quad 0^{\circ} \quad 10^{\circ} \mathrm{E} \quad 20^{\circ} \mathrm{E} \quad 30^{\circ} \mathrm{E}$

SOA, Biomass burning, summer

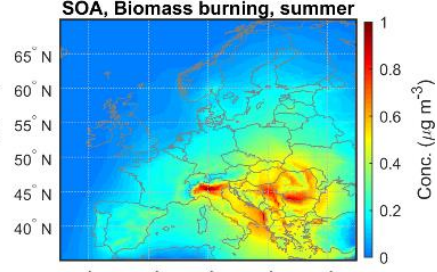

$10^{\circ} \mathrm{W} \quad 0^{\circ} \quad 10^{\circ} \mathrm{E} \quad 20^{\circ} \mathrm{E} \quad 30^{\circ} \mathrm{E}$
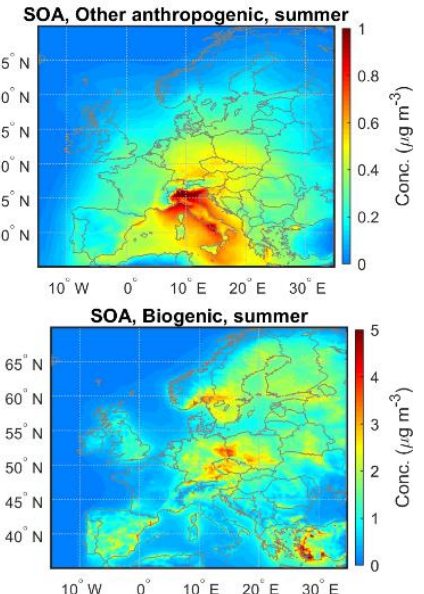

Figure S4: Spatial distributions of primary and secondary OA from different sources in winter (a, b) and summer (c, d). The winter and summer results are the averages of December - January - February and June - July - August, respectively. Note

5 that different scales are used for biomass burning and biogenic source to facilitate visualization. 

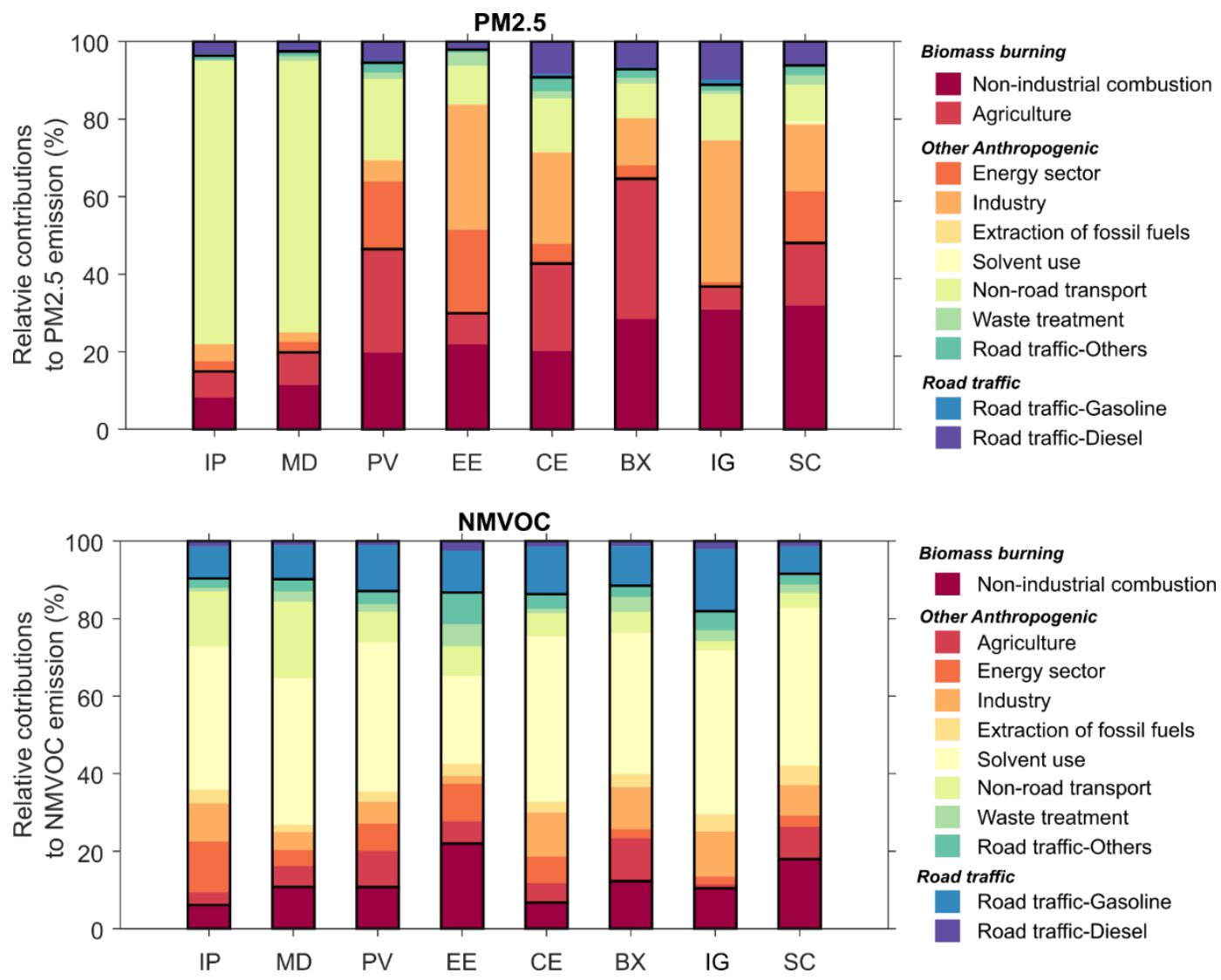

Figure S5: Relative contributions of different anthropogenic sources to total $\mathrm{PM}_{2.5}$ and NMVOC emissions in 2011. The 8 sub-regions are the Iberian Peninsula (IP), the Mediterranean (MD), Po Valley (PV), eastern Europe (EE), central Europe 5 (CE), Benelux (BX), Ireland and Great Britain (IG), and Scandinavia (SC). 